\title{
La aplicación de modelos de distribución de especies para la realización de inferencias arqueológicas. Una ejemplificación a partir de Geoffroea decorticans en el área Sudcalchaquí (Noroeste, Argentina)
}

\author{
Francisco Franco y Gonzalo Andrés Camps \\ Recibido 03 de noviembre 2019. Aceptado 06 de marzo 2020
}

\section{RESUMEN}

En este aporte interdisciplinario se desarrolla el concepto de modelos de distribución de especies, sus implicancias y las estrategias utilizadas para inferirlos. Se los ejemplifica a partir de la aplicación de la herramienta MaxEnt para modelar la distribución del árbol conocido como chañar (Geoffroea decorticans (Gillies ex Hook. \& Arn.) Burkart) entre 4200 y 300 años AP. Finalmente, se plantean implicancias y potencialidades arqueológicas generadas a partir del uso del modelado de distribución para distintos sitios arqueológicos del área Sudcalchaquí, en los cuales se ha identificado el consumo de la especie durante el primer milenio d.C.

Palabras clave: Modelado de distribución de especies; Geoffroea decorticans; Movilidad/Circulación de recursos; Paleoclima.

\begin{abstract}
SPECIES DISTRIBUTION MODELING AS A TOOL FOR INFERENCE: AN EXAMPLE FROM GEOFFROEA DECORTICANS IN THE SOUTHERN CALCHAQUI AREA, NORTHWESTERN ARGENTINA. In this interdisciplinary contribution, first, the concept of species distribution modeling is developed along with its implications, potential, and inferential strategies. Second, application of the MaxEnt tool is demonstrated based on the distribution modeling of the chañar tree (Geoffroea decorticans (Gillies ex Hook. \& Arn.) Burkart)) between 4200 and 300 BP. Third, archaeological implications are generated from the use of distribution modeling for different archaeological sites in Northwest Argentina where the consumption of Geoffroea decorticans during the first millennium A.D. has been identified.
\end{abstract}

Keywords: Species distribution modeling; Geoffroea Decorticans; Mobility/Circulation of resources; Paleoclimate.

\section{INTRODUCCIÓN}

En este trabajo se desarrolla el uso de Modelos de Distribución de Especies (MDE) a partir de la herramienta MaxEnt y su aplicación a problemáticas arqueológicas. Se considera que esta herramienta probabilística -habitualmente utilizada por diversas ramas de la biología y la medicina (Elith y
Leathwick, 2009; Peterson et al., 2011; AlvaradoSerrano y Knowles, 2014)- también puede aplicarse para inferir dinámicas sociales a nivel arqueológico en relación con la utilización o circulación de recursos naturales vegetales y/o animales.

A pesar de su potencial para evaluar posibles interacciones entre humanos y recursos biológicos, y en particular sobre procesos de consumo y movilidad, MaxEnt ha sido escasamente aplicado por

Francisco Franco. Instituto de Estudios Históricos (IEH). Consejo Nacional de Investigaciones Científicas y Técnicas (CONICET). Corro 308 (CP 5000). Córdoba, Argentina. E-mail: franfranco@unc.edu.ar

Gonzalo Andrés Camps. Instituto Multidisciplinario de Biología Vegetal (IMBIV). Consejo Nacional de Investigaciones Científicas y Técnicas (CONICET). Av. Vélez Sársfield I6II (X50I6GCA), Córdoba, Argentina. E-mail: campsandres@gmail.com 
la arqueología. En Argentina, solo recientemente ha comenzado a utilizarse para modelar paleodistribuciones de recursos de caza (Politis et al., 2011) y de preferencias ambientales de la entidad arqueológica Goya-Malabrigo (Politis et al., 2018).

En este artículo, ampliamos su horizonte de aplicación mediante un ejercicio que permite demostrar su potencial analítico en cuanto a la circulación de recursos vegetales. Para ello se aborda el concepto de MDE, sus implicancias, y las estrategias utilizadas para inferirlos. Luego se ejemplifica a partir de la aplicación de MaxEnt para modelar la distribución de chañar (Geoffroea decorticans [(Gillies ex Hook. \& Arn.) Burkart]) entre 4200 y 300 años AP. Finalmente, se plantean implicancias y potencialidades arqueológicas generadas a partir del uso del modelo de distribución de chañar para distintos sitios arqueológicos del área Sudcalchaquí, en los cuales se ha identificado el consumo de esta especie vegetal durante el primer milenio d.C. ${ }^{1}$

\section{Modelos de Distribución de Especies: conceptualización y alcances}

Los MDE permiten aproximarse al conjunto de condiciones climáticas en las que una especie logra sobrevivir (Peterson et al., 2011); estas se pueden proyectar en el espacio geográfico, lo cual posibilita adquirir una noción del área de distribución potencial de la especie de interés (Elith y Leathwick, 2009).

A nivel teórico, los MDE se constituyen como representaciones geoespaciales del diagrama BAM desarrollado por Soberón y Peterson (2005). Estos autores plantean que el área de distribución de una especie es el resultado de una combinación de factores, en particular tres: 1) B: la región del mundo que contiene el ambiente biótico adecuado para la especie; 2) A: la región del mundo donde se encuentran las condiciones ambientales que permiten la sobrevivencia y reproducción de la especie; y 3) $\mathrm{M}$ : el conjunto de sitios que la especie ha explorado; $M$ es, de alguna manera, su capacidad de dispersión por el espacio.

La potencia inferencial de un MDE dependerá del tipo y la calidad de datos ingresados, siendo los de primer orden aquellos concernientes a los puntos de presencia (puntos en el espacio geográfico donde la especie de interés está presente), y posteriormente los datos climáticos y ambientales. Una herramienta de creciente utilización para la realización de modelados es MaxEnt (Phillips et al., 2006), la cual se enfoca en ajustar una distribución de probabilidad para la ocurrencia de la especie de interés al conjunto de píxeles en la región de estudio, basándose en la idea de que la mejor explicación a los fenómenos desconocidos maximizará la entropía de la distribución de probabilidad, sujeto a los valores actuales allí donde se encuentra la especie (Phillips et al., 2004; Peterson et al., 2007). Es decir, genera un MDE que, en función de las características climáticas de cada punto de un espacio geográfico reticulado, asigna una determinada probabilidad de existencia de la especie en esa ubicación.

Los MDE asumen que el nicho de la especie en estudio se conserva (Peterson et al., 2011); bajo ese supuesto, busca las mismas condiciones ambientales que resultaron al modelar el clima presente y las transfiere a un escenario climático pasado o futuro (Peterson et al., 2011). El resultado es una estimación de posibles áreas donde la especie podría haberse establecido en el período de tiempo transferido. $^{2}$

\section{Geoffroea decorticans, nomadismo/ sedentarismo y circulación de recursos silvestres}

Geoffroea decorticans es un árbol pequeño a mediano (de 6 a 10 metros de altura), pionero en la sucesión y de amplia tolerancia al frío y la sequía. En Argentina, se distribuye principalmente en los pisos ecológicos chaco húmedo y semiárido, espinal, prepuna y yungas (Demaio et al., 2002). En la región chaqueña, también se observa la presencia de otra especie de su género, Geoffroea spinosa; ambas son las únicas representantes nativas del género en Sudamérica (Ireland y Pennington, 1999). Los frutos del chañar, de elevado valor nutricional (100 g/kg de proteína bruta [Orrabalis et al., 2013]), pueden consumirse frescos y/o secos, o también ser utilizados para la elaboración de harina, arrope y bebidas alcohólicas (Storni, 1942; Demaio et al., 2002; Calo, 2010); la corteza ha sido tradicionalmente utilizada con fines medicinales y/o tintóreos (Besio, 2017) y su madera es utilizada como leña (Demaio et al., 2002) (Figura 1).

Al considerar la amplia cantidad de sitios arqueológicos correspondientes a múltiples temporalidades y espacialidades en los que se ha identificado el uso de Geoffroea decorticans, se la debe abordar como una especie transversal en las lógicas 


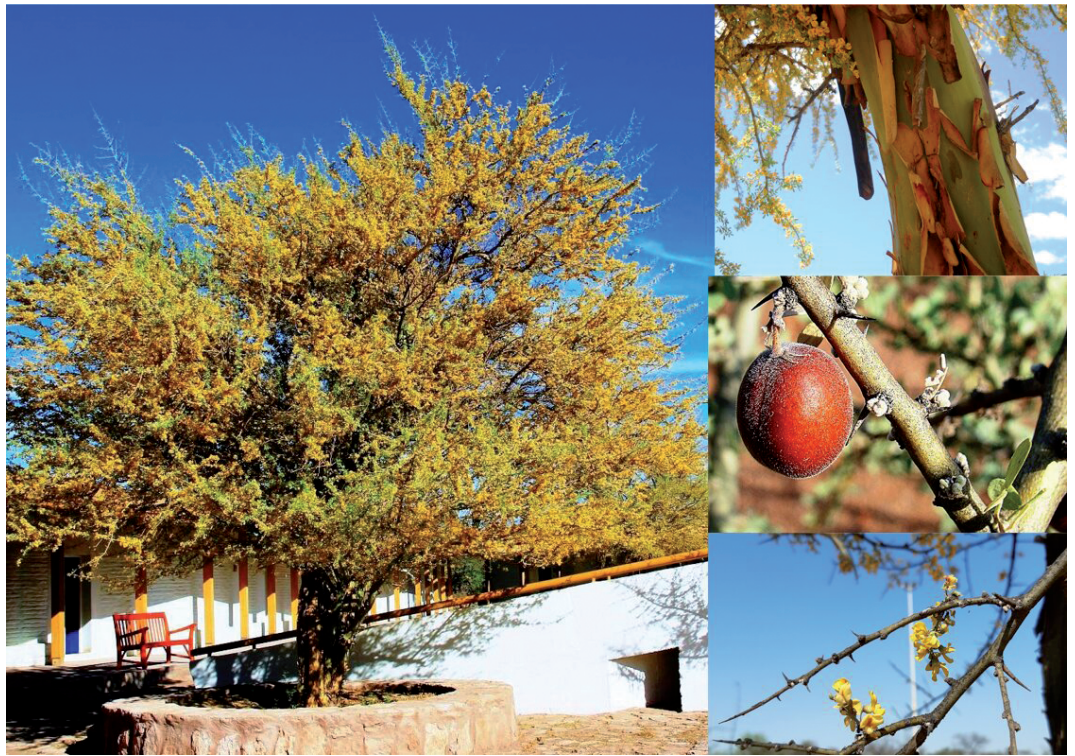

Figura 1. Ejemplar de chañar y detalle de corteza, fruto y flor. Fotografías de M. Mercadante, D. Culbert y F. Franco.

de articulación y reproducción social de los grupos prehispánicos. Sus cualidades intrínsecas también tienen implicancias de relevancia. En primer lugar, el crecimiento natural en distintos pisos ecológicos implica una distribución amplia en el NOA, pero no total, cuya evidencia de uso en algunos sectores remite a lógicas de movilidad/circulación de personas en diversas escalas; en segundo término, la perennidad ha sido destacada como una condición que implica vinculaciones profundas y recurrentes en la relación humanos-plantas-espacio (Besio, 2017); por último, la estacionalidad de los frutos presentes en los meses estivales marca una temporalidad cíclica para la posibilidad de recolección, la cual ha sido reseñada etnohistóricamente como una época de grandes festejos y borracheras (Lorandi y Boixados, 1988; Noli, 1998; Farberman, 2006).

Geoffroea decorticans es una especie que se puede pensar desde dos vertientes críticas desarroIladas recientemente en la arqueología argentina, ya que se insertaría dentro de esquemas de movilidad/ circulación de grupos caracterizados tradicionalmente por su sedentarismo; y también debido a su importancia social en grupos con marcado desarroIlo agrícola. En este sentido, se considera que las categorizaciones movilidad/sedentarismo y producción/extracción, conceptualizadas como pares dicotómicos, no resultan operativas para desentramar la pluralidad de estrategias de subsistencia y de movilidad en el tiempo y el espacio (Lema, 2008; Ortiz y Killian Galván, 2016). El caso de las especies arbóreas de recolección se ubica en el centro de estos cuestionamientos, y permite un abordaje que complejiza las lógicas desarroIladas desde narrativas lineales.

En primer lugar, las categorías de nomadismo y sedentarismo han sido replanteadas desde hace décadas en la arqueología internacional ya que las prácticas de movilidad son inherentes a todos los grupos sociales en distintas formas y escalas, y no necesariamente correlativas con un determinado modo de subsistencia (Kelly, 1992; Rocek, 1996). Estos postulados han comenzado a tener un correlato en el creciente corpus de investigaciones sobre arqueología del tráfico de personas, animales y bienes en el NOA, las cuales han aportado valiosa información sobre estrategias de movilidad y circulación de recursos en contextos tradicionalmente interpretados como sedentarios (e.g., Lazzari, 2010; Martel et al., 2017).

En segundo lugar, la perspectiva lineal que implica la transición de la extracción a la producción también ha sido motivo de críticas, cuyos fundamentos derivan del registro de grupos catalogados como cazadores-recolectores que han experimentado la producción de recursos, y también de sociedades agropastoriles del NOA que evidenciaron elevados consumos de recursos silvestres (Aschero y Hocsman, 2011; Babot, 2011, entre otros).

En relación con las prácticas de recolección, investigaciones recientes han posibilitado determinar con un mayor grado de certeza la pregunta: ¿qué consumían las sociedades prehispánicas del NOA? Estas identificaron una amplia cantidad de recursos, incluyendo el aprovechamiento de numerosas especies silvestres a lo largo de todo el Holoceno tardío (Yacobaccio y Korstanje, 2007; Lema, 2008; Aschero y Hocsman, 2011; Lema et al., 2012, entre otros). Sin embargo, quedan aún por desarrollar una serie de interrogantes más amplios en relación con la proveniencia de los recursos y/o las interacciones establecidas para su procuramiento.

En lo respectivo a la distribución de las especies, las diferencias fitogeográficas entre distintos sectores del NOA han sido utilizadas para realizar analogías en base a la observación actual. Sin embargo, las diferencias paleoclimáticas y la acción humana durante distintos momentos históricos implican que la 
extrapolación actualística de distribución de especies no sea necesariamente correlativa con la que existiera en tiempos prehispánicos.

El grado de resolución espacial obtenido analógicamente puede ser perfeccionado a partir de herramientas probabilísticas como el MDE, la cual permite superar parcialmente las vicisitudes de la analogía a partir de indicadores estadísticos, lo que facilita el desarrollo de hipótesis de proveniencia y distancia potencial de los recursos. En este caso, la selección de los sitios para la modelización se basó en la necesidad de evaluar posibles sectores de procuramiento de un registro inédito de chañar en Mortero Quebrado (valle de Anfama, Tucumán), debido a que actualmente no se observaba la presencia de la especie en el área.

Mortero Quebrado (MQ) es un conjunto de siete unidades residenciales dispersas (2300 msnm), las cuales, en base a tres dataciones radiocarbónicas, han sido ubicadas cronológicamente entre 1800 y 1500 AP. La arquitectura de las viviendas consiste en grandes patios centrales de planta circular (10 metros de diámetro aproximadamente), delimitados por muros, a los cuales se adosan recintos habitacionales de la misma morfología (2 a 3 metros de diámetro), similares a los observados en los vecinos valles de Tafí y La Ciénega (Oliszewski, 2017). La cultura material identificada consiste en cerámica utilitaria, instrumentos líticos (entre los cuales destacan obsidianas de la fuente Ona-Las Cuevas), y wankas formatizadas (Salazar y Molar, 2017). En función del patrón arquitectónico y material de $\mathrm{MQ}$, se considera que se puede integrar al sitio dentro de los esquemas de circulación de materiales e ideas del área sudcalchaquí durante el primer milenio de la Era (Scattolin, 2007; Lazzari, 2010; Oliszewski, 2017, entre otros).

\section{METODOLOGÍA}

\section{Presencia de Geoffroea decorticans en sitios del primer milenio d.C. en el área Sudcalchaquí}

La necesidad de evaluar posibles sectores de origen de chañar en MQ implicó la reflexión sobre otras ocupaciones relativamente contemporáneas (primer milenio d.C.) de la región en donde se hubiera utilizado la especie. Disponer de hipótesis iniciales implicó integrar los registros en un MDE, que permitiera evaluar posibles sectores de procuración o circulación de Geoffroea decorticans entre estas poblaciones. En función de ello, se consideraron sitios arqueológicos que, por proximidad, temporalidad y/o cultura material asociada, podrían integrarse dentro de un esquema de interrelación regional, y donde las investigaciones hayan planteado la utilización antrópica del chañar.

Los sitios escogidos en dirección este a oeste y el respectivo indicador de la especie fueron: Mortero Quebrado -leño carbonizado- (valle de Anfama); Yánimas 1 -indeterminado en la publicación original- (piedemonte sur de Tucumán); La Bolsa 1 -endocarpos- y Casas Viejas -endocarpos(valle de Tafí); Puesto Viejo 2 -endocarpos- (quebrada de Los Corrales); Los Colorados -semillas-, Soria 2 -semillas-, y Tesoro 1 -endocarpo- (valle de Yocavil); Cardonal -endocarpos y semillas- (valle del Cajón); Los Viscos -endocarpos- (valle de El Bolsón); y Punta de las Peñas 9 -semilla- (puna).

Los registros en las ocupaciones seleccionadas se encuentran íntegramente publicados (Pochettino y Scattolin, 1991; Carrizo et al., 1997; Korstanje y Wurschmidt, 1999; López Campeny y Escola, 2007; Calo, 2010; Molar, 2014; Miguez y Caria, 2015; Oliszewski et al., 2015; Palamarczuk et al., 2016; Petrucci y Spano, 2019), a excepción de Mortero Quebrado, el cual se presenta aquí por primera vez. A partir de la comparación de la distancia entre los sitios y el MNE se realizó una primera clasificación entre proveniencia local -hasta $8 \mathrm{~km}$-, regional -entre 8 y $35 \mathrm{~km}-$, y de larga distancia -más de 35 km- (Lynch y Rocek, 2019).

\section{Aplicación del modelo de distribución para Geoffroea decorticans}

La aplicación de un MDE para Geoffroea decorticans consideró los registros climáticos actuales (promedio de los años 1979-2013), luego de lo cual se extrapoló a un escenario paleoclimático (promedio de los años 4200-300 AP).

La base de datos para el clima actual se obtuvo de CHELSA (Karger et al., 2017a; disponible en Karger et al., 2017b), y la base de datos para tiempo histórico se obtuvo de PaleoClim (Fordham et al., 2017; Brown et al., 2018), ${ }^{3}$ ambas con resolución de 2.5 arc-min (reticulado espacial de $5 \mathrm{~km}^{2}$ ). Las bases son equivalentes dado que tienen la misma información (datos de temperatura y precipitación), a partir de la cual se calculan promedios anuales, trimestrales, mensuales y rangos, que dan lugar a 19 
variables bioclimáticas (las variables pueden consultarse en Karger et al. [2017a] y Fordham et al. [2017]). El clima promedio del Holoceno tardío se recupera del TRaCE21 ka experiment, el cual evalúa: concentración de gases de efecto invernadero de larga pervivencia, evolución de capas de hielo de los glaciares y contribución de agua glaciar a los océanos; para proyectar condiciones paleoclimáticas con una elevada precisión (Fordham et al., 2017). El modelado en tiempo actual se utilizó a fines comparativos, ya que permite evaluar la divergencia entre ambos períodos. En tanto, el uso del promedio climático de los años 4200-300 AP (Holoceno tardío, Meghalayan) ${ }^{4}$ ha sido utilizado para evaluar la distribución potencial en tiempos prehispánicos.

Al respecto, Anfama no cuenta con antecedentes paleoclimáticos, pero sí se han desarrollado estudios en sectores vecinos. En el piedemonte oriental, Gómez Augier y Caria (2012) -en base a análisis paleoedáficos, paleogeomorfológicos, sedimentológicos y de microfósiles- dan cuenta de condiciones de mayor aridez entre 4000 y 2500 AP; seguidas por otras relativamente húmedas entre 2500 y 1200 AP aproximadamente; cálidas y secas entre 1200 a 800 AP, a partir de lo cual comenzaría una etapa más fría pero también seca hasta 200 AP. Los mismos autores plantean que en los valles de Tafí y Yocavil, hacia 4200 AP, habrían surgido condiciones más secas que las actuales, situación que se habría revertido entre 2300 y 1200 AP y que habría terminado hacia $1100-$ 800 AP, momento en el que habría comenzado un período de acentuadas sequías. En tanto, en el valle de El Bolsón se ha marcado un proceso de mayor humedad que la actual entre 4200 a 2800 AP, seguido por un máximo de humedad entre
2800 y 1400-1500 AP, continuado por un lapso de mayor aridez y menor temperatura que el precedente (Kulemeyer et al., 2013). Por último, para el sector puneño de Antofagasta de la Sierra se ha planteado el comienzo de una fase de mayor humedad en relación con el Holoceno medio a partir de 4200-3800 AP, que se consolida en el lapso 3500-3000 AP. Esa fase húmeda se extendería hasta 1600-1400 AP, lapso a partir del cual comienza una nueva fase de marcada aridez hasta 600-300 AP (Grana et al., 2016).

Sintéticamente, la región considerada habría tenido condiciones relativamente más húmedas que las actuales (4200 AP hasta 2800-2500 AP); continuadas por un período aún de mayor humedad, conocido habitualmente como "período húmedo del Holoceno tardío" (2800-2500 AP hasta 1400-1200 AP aproximadamente); luego seguido por otro de altas temperaturas y seco conocido como "cálido medieval" (800-600 AP hasta 300 AP aproximadamente [Gómez Augier y Caria, 2012]).

El MDE en tiempo actual se realizó a partir de puntos de ocurrencia de la especie, obtenidos de Global Biodiversity Information Facility. ${ }^{5}$ Se seleccionaron los puntos de ocurrencia más recientes (1985 a 2013) con georreferenciación precisa. Para evitar sesgos predictivos (mayor representación de una condición ambiental), se eliminaron puntos
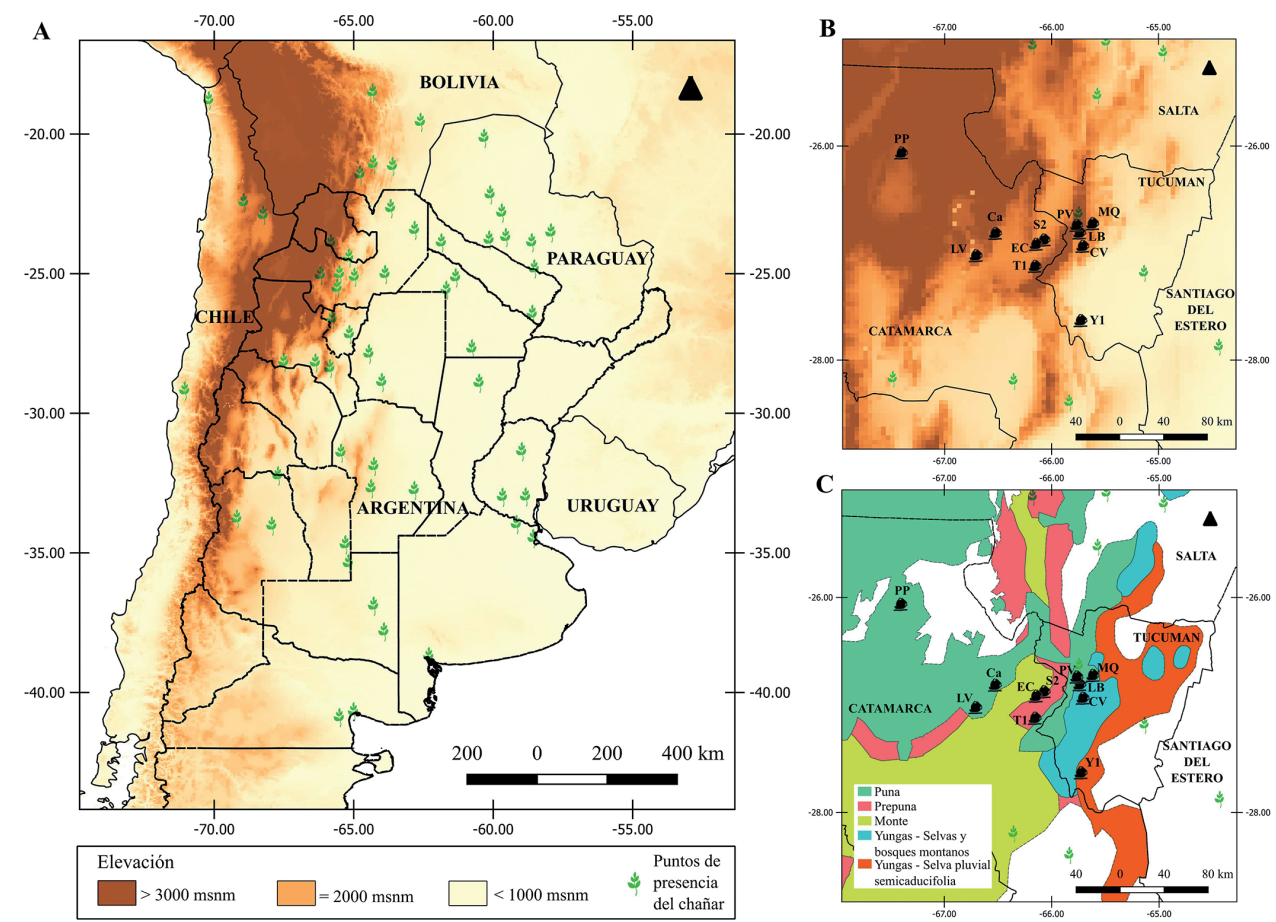

Figura 2. A) Puntos de presencia actuales de Geoffroea decorticans. B) Mapa integrado de altimetría, puntos de presencia y sitios arqueológicos considerados para un sector del NOA. C) Mapa integrado de pisos ecológicos, puntos de presencia y sitios arqueológicos considerados para un sector del NOA. Pisos ecológicos de acuerdo con Oyarzabal et al. (2018). 
duplicados y cercanos en un radio de $0.5^{\circ}$. De los 85 puntos de ocurrencia (Figura 2) que cumplieron estos requisitos, el $70 \%$ (58 puntos) se usó para calibrar el modelo y el 30\% (27 puntos) para validarlo.

Luego se estimó la capacidad de dispersión de la especie (área M sensu diagrama BAM), siguiendo un criterio biogeográfico, bajo la hipótesis de que la especie puede distribuirse en la ecorregión actual y las contiguas. Las 19 variables bioclimáticas del tiempo actual obtenidas de CHELSA fueron recortadas al tamaño del área $\mathrm{M}$ y se eliminaron aquellas que estaban fuertemente correlacionadas (Pearson $>0,85)$. En un segundo paso de selección, se descartaron también las que presentaban una ganancia menor visualizada en la importancia de permutación y en la prueba Jackknife.

Una vez seleccionados los puntos y las variables bioclimáticas, el MDE por defecto para la especie en MaxEnt fue evaluado con la librería en $\mathrm{R}$ ENMeval (Muscarella et al., 2017). Esto permitió probar un conjunto de variables predictoras ("feature classes", Fc) junto con diferentes valores de penalización por complejizar el modelo ("regularization multiplier", Rm), lo que dio como resultado 48 modelos diferentes de MaxEnt, de los cuales se eligieron los cuatro con los valores más bajos del criterio de información de Akaike corregido (AICc) y tasa de omisión más baja (datos de presencia real de la especie que fueron clasificados como ausencia por el modelo). Posteriormente, los cuatro modelos se validaron con las herramientas provistas en Niche Tool Box (Osorio-Olvera et al., 2018) utilizando ROC parcial (Peterson et al., 2008), prueba binomial (Anderson et al., 2002), error de omisión (falso negativo de Fielding y Bell, 1997), "true skill statistic" (TSS) (Allouche et al., 2006), Kappa (Cohen, 1960) y la relación puntos de presencia no predichos/superficie predicha $\left(\mathrm{km}^{2}\right)$ (Camps et al., 2018), con el objetivo de seleccionar el mejor de los cuatro. La validación se realizó con el 30\% de puntos de presencias apartados inicialmente y seis puntos de ausencias conocidas de la especie.

El MDE en tiempo actual se transfirió al escenario climático representativo del Holoceno tardío, utilizando las mismas variables bioclimáticas y parámetros seleccionados en el paso anterior. De esta manera, se trasladan al pasado las preferencias climáticas actuales de la especie, ya que son las condiciones conocidas en las que habita. Para probar si las áreas proyectadas al pasado se detectaron como extrapolaciones estrictas o áreas con similitud ambiental entre las regiones de calibración -MDE actual-y proyección -MDE tiempo pasado- (Owens et al., 2013), se utilizó el análisis de superficie de similitud ambiental multivariante (MESS; Elith et al., 2010), implementado en MaxEnt. Para la construcción de los mapas y figuras se utilizó Quantum GIS 2.18 e Inkscape 0.91 .

\section{RESULTADOS}

En primer lugar, los carbones obtenidos en los pisos de ocupación de dos de las unidades residenciales de MQ fueron analizados para identificar las especies vegetales allí utilizadas. La observación fue realizada por la Lic. Verónica Mors en microscopio óptico a 40X/200X de aumento. Los caracteres cualitativos se compararon con atlas anatómicos de maderas (Tortorelli, 1956) y con ejemplares actuales de referencia ubicados en la colección del Museo de Antropología (Córdoba). Los resultados constataron la presencia de Geoffroea decorticans carbonizado en ambos pisos de ocupación (Figura $3)$ aunque en baja frecuencia $(0,82 \%)$ en relación con la muestra considerada $(\mathrm{N}=244)$.

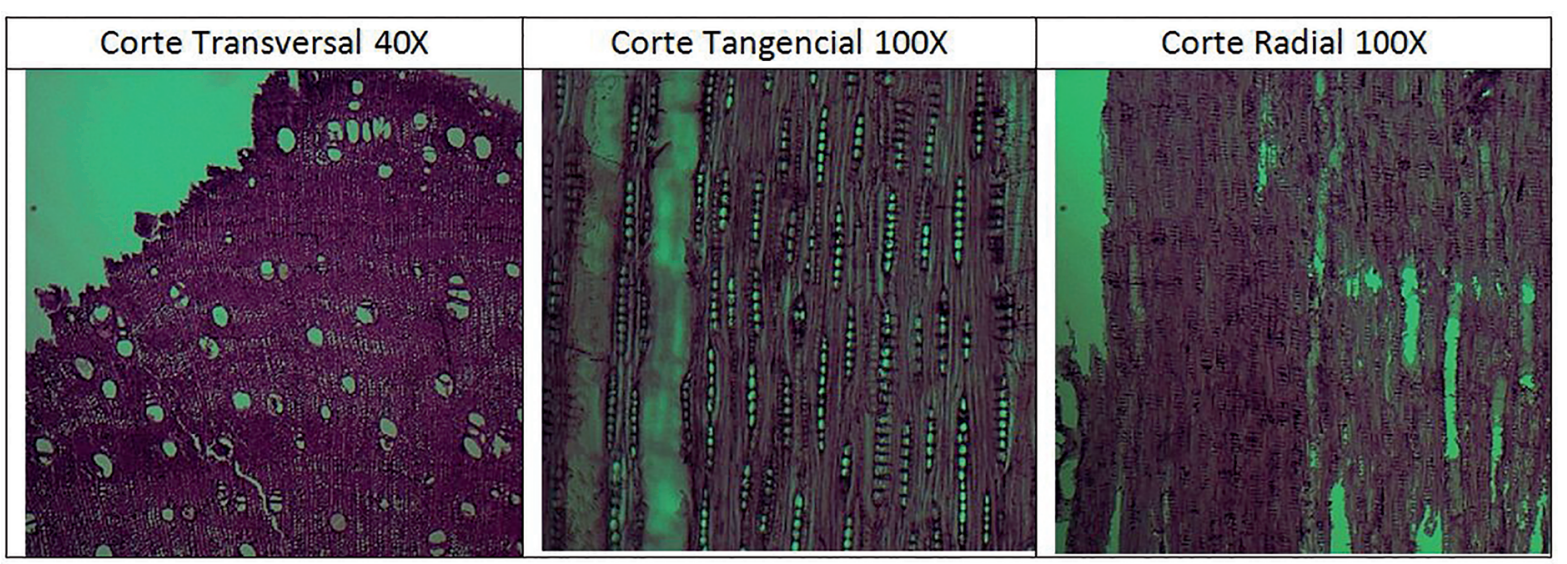

Figura 3. Detalle microscópico de Geoffroea decorticans recuperado en Mortero Quebrado. 
En segundo lugar, los cuatro modelos más robustos en tiempo presente de MaxEnt que resultaron al aleatorizar los parámetros se validaron para seleccionar al mejor de ellos. De acuerdo con la Tabla 1, que contiene las métricas de validación, el modelo 3 (parámetros $F_{C}$ : LQHPT, rm: 3 ) es el que posee, en general, los mejores indicadores. El modelo 3 presenta el segundo valor más alto de ROC parcial pero contiene, junto con el modelo 4, los valores más altos en las métricas que resumen el poder de predicción del modelo (Kappa, TSS y error de omisión). A su vez, los modelos 3 y 4 tienen la misma cantidad de fallas en la predicción de presencias (6), pero el modelo 3 es más predictivo, ya que las presenta en un espacio geográfico más reducido. El análisis MESS, que valida las proyecciones del modelo transferido, dio como resultado que el ambiente de transferencia (pasado) no presenta variables climáticas fuera del rango de lo observado en el ambiente de calibración (presente); es decir, las predicciones son confiables, ya que el algoritmo no asigna valores de probabilidad basado en rangos ambientales que en la actualidad no existen. De las variables utilizadas, las siguientes realizaron un mayor porcentaje de aporte en la resolución del modelo: temperatura media del trimestre más húmedo $(45,8 \%)$, temperatura media del trimestre más seco $(12,2 \%)$, precipitación del trimestre más frío $(9,2 \%)$ y precipitación del trimestre más cálido $(9,1 \%)$.

Los resultados obtenidos del modelo 3 permitieron generar dos mapas probabilísticos (i.e., tiempos presente y pasado; Figura 4), cuyos colores oscilan en una escala entre blanco (probabilidad de ocurrencia menor al umbral establecido del 10\%) y rojo (probabilidad baja, de 0,38-0,52\%; probabilidad media, de 0,52-0,65\%; y probabilidad alta, de $0,66-0,8 \%$ ).

\section{DISCUSIÓN}

La observación de ambos MDE permite plantear en primer lugar que la comparación diacrónica entre el modelado a clima actual y el realizado con paleoclima (Figura 4 y Tabla 2) estima que las condiciones para el desarrollo de chañar en el área sudcalchaquí son levemente más propicias en la actualidad. Esto se observa principalmente en la alta probabilidad de ocurrencia presente en amplios sectores del norte de Catamarca, centro-este de Tucumán y sur de Salta. Algunos sectores hacia el sudeste de Antofagasta de la Sierra también presentan actualmente condiciones que permitirían desarrollar la especie, situación que no se observa durante el Holoceno tardío.

En el caso del modelado paleoclimático, se observa que, si bien el patrón de distribución de la especie pudo haber sido similar, la presencia habría sido menor durante el Holoceno tardío. En este sentido, la realización de analogías en base a la observación actual puede correr el riesgo de sobrerrepresentar la presencia pretérita de la especie.

\begin{tabular}{|l|l|l|l|l|}
\hline \multicolumn{1}{|c|}{$\begin{array}{c}\text { Métricas de } \\
\text { validación }\end{array}$} & $\begin{array}{c}\text { Modelo 1 } \\
\text { (LQHP 3.5) }\end{array}$ & $\begin{array}{c}\text { Modelo 2 } \\
\text { (LQHPT 3.5) }\end{array}$ & $\begin{array}{c}\text { Modelo 3 } \\
\text { (LQHPT 3) }\end{array}$ & $\begin{array}{c}\text { Modelo 4 } \\
\text { (LQHP 3) }\end{array}$ \\
\hline ROC parcial & 1,43 & 1,40 & 1,41 & 1,37 \\
\hline Kappa & 0,36 & 0,36 & 0,41 & 0,41 \\
\hline TSS & 0,73 & 0,73 & 0,77 & 0,77 \\
\hline Error de omisión & 0,27 & 0,27 & 0,23 & 0,23 \\
\hline Test binomial & 0,029 & 0,029 & 0,009 & 0,009 \\
\hline $\begin{array}{l}\text { Superficie predicha (km²) } \\
\text { (L) }\end{array}$ & $2.205,486$ & 2.184 .471 & 2.222 .801 & 2.393 .120 \\
\hline Presencias no predichas & 7 & 7 & 6 & 6 \\
\hline $\begin{array}{l}\text { Relación (superficie/ } \\
\text { presencias no predichas) } \\
* 100\end{array}$ & 0,000317 & 0,000320 & 0,000270 & 0,000251 \\
\hline Umbral (10\%) & 0,379 & 0,377 & 0,362 & 0,336 \\
\hline
\end{tabular}

*Por debajo de este valor, que representa la exclusión del 10\% de los puntos con probabilidad más baja en cada modelo, no se considera una probabilidad aceptable para ser reflejada en el mapeo del MDE.

Tabla 1. Métricas de validación para los cuatro mejores modelos de MaxEnt obtenidos. Se detalla entre paréntesis la configuración de parámetros Fc y rm que resultó para cada uno.

En segundo lugar, de la comparación entre el MDE paleoclimático y los sitios del primer milenio d.C. con evidencias de uso de Geoffroea decorticans también se pueden plantear hipótesis de interés. La más evidente -ya planteada por López Campeny y Escola (2007)- es la circulación a larga distancia de chañar en Punta de las Peñas. Los puntos de proveniencia más cercanos se encontrarían, cuanto menos, a unos $100 \mathrm{~km}$ de distancia.

Sitios como Puesto Viejo 2 y Tesoro 1 también 


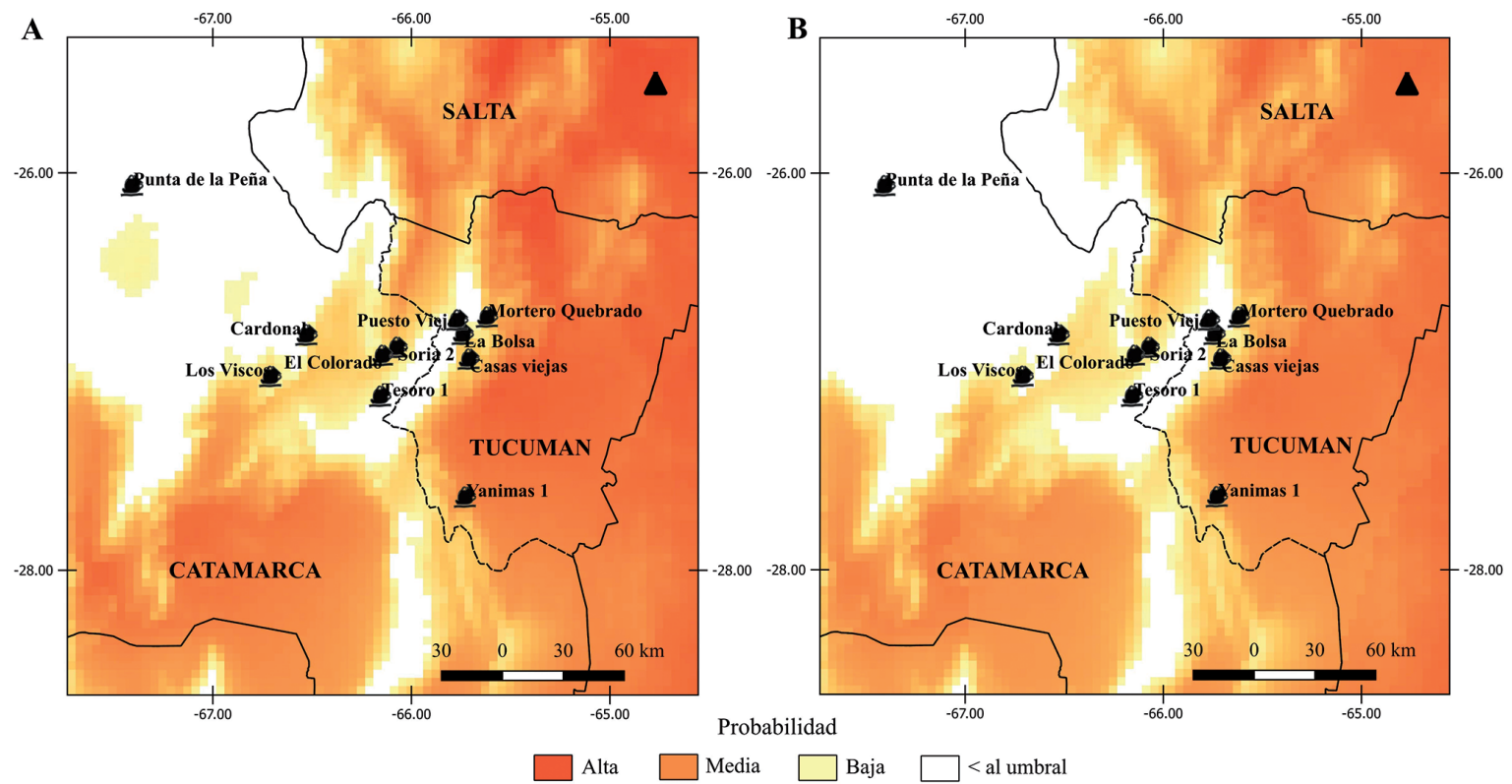

Figura 4. Modelo de distribución de Geoffroea decorticans proyectado al espacio geográfico para el NOA. A) MDE promedio para tiempo actual (1979-2013). B) MDE para el Holoceno tardío (4200-300 AP). Se muestran los sitios arqueológicos considerados.

se encuentran por fuera de áreas con probabilidad de chañares (Tabla 2), avalados además por la altimetría (mayor a $3000 \mathrm{msnm}$ ), ya que no se cuenta con evidencia de crecimiento de la especie a tal elevación; su procuramiento se habría realizado desde sectores relativamente próximos (8 a $15 \mathrm{~km}$ de distancia aproximada) de carácter regional.

\begin{tabular}{|c|c|c|c|c|c|c|}
\hline Sitio & Provincia & $\begin{array}{l}\text { Ubicación } \\
\text { geográfica }\end{array}$ & $\begin{array}{c}\text { Región } \\
\text { fitogeográfica }\end{array}$ & $\begin{array}{l}\text { Altura } \\
\text { aprox. } \\
\text { (msnm) }\end{array}$ & $\begin{array}{c}\text { Probabilidad } \\
\text { presencia } \\
\text { actual }\end{array}$ & $\begin{array}{c}\text { Probabilidad } \\
\text { presencia } \\
\text { Holoceno }\end{array}$ \\
\hline $\begin{array}{l}\text { Mortero } \\
\text { Quebrado }\end{array}$ & Tucumán & $\begin{array}{l}\text { Valle de } \\
\text { Anfama }\end{array}$ & $\begin{array}{l}\text { Yungas (Bosque } \\
\text { montano) }\end{array}$ & 2200 & 0,485 & 0,453 \\
\hline Yánimas 1 & Tucumán & $\begin{array}{l}\text { Faldeo oriental } \\
\text { del Aconquija }\end{array}$ & $\begin{array}{l}\text { Yungas (Selva pluvial } \\
\text { semicaducifolia) }\end{array}$ & 750 & 0,578 & 0,547 \\
\hline Casas Viejas & Tucumán & Valle de Tafí & $\begin{array}{l}\text { Yungas (Bosque } \\
\text { montano) }\end{array}$ & 1900 & 0,496 & 0,465 \\
\hline La Bolsa 1 & Tucumán & Valle de Tafí & $\begin{array}{l}\text { Valle intermontano } \\
\text { (Queshua) }\end{array}$ & 2500 & 0,424 & 0,396 \\
\hline $\begin{array}{l}\text { Taller Puesto } \\
\text { Viejo }\end{array}$ & Tucumán & $\begin{array}{l}\text { Quebrada del } \\
\text { Infiernillo }\end{array}$ & Prepuna & 3100 & 0,371 & 0,348 \\
\hline Soria 2 & Catamarca & $\begin{array}{c}\text { Valle } \\
\text { de Yocavil }\end{array}$ & $\begin{array}{l}\text { Valle intermontano } \\
\text { (Queshua) }\end{array}$ & 2150 & 0,474 & 0,445 \\
\hline El Colorado & Catamarca & $\begin{array}{c}\text { Valle } \\
\text { de Yocavil }\end{array}$ & $\begin{array}{l}\text { Valle intermontano } \\
\text { (Queshua) }\end{array}$ & 2150 & 0,491 & 0,456 \\
\hline Tesoro 1 & Catamarca & Valle de Yocavil & Prepuna & 3000 & 0,378 & 0,356 \\
\hline Cardonal & Catamarca & $\begin{array}{c}\text { Valle } \\
\text { del Cajón }\end{array}$ & $\begin{array}{l}\text { Valle intermontano } \\
\text { (Queshua) }\end{array}$ & 2800 & 0,395 & 0,378 \\
\hline Los Viscos & Catamarca & $\begin{array}{l}\text { Valle de } \\
\text { El Bolsón }\end{array}$ & $\begin{array}{c}\text { Valle intermontano } \\
\text { (Queshua) }\end{array}$ & 2750 & 0,426 & 0,396 \\
\hline $\begin{array}{l}\text { Punta de la } \\
\text { Peña } 9\end{array}$ & Catamarca & $\begin{array}{l}\text { Antofagasta } \\
\text { de la Sierra }\end{array}$ & Puna & 3350 & 0,326 & 0,287 \\
\hline
\end{tabular}

Tabla 2. Sitios arqueológicos considerados, valores de probabilidad para cada escenario e inferencia sobre origen del chañar en cada sitio. Se marcan en negrita los valores de probabilidad que se encuentran por debajo del umbral considerado para cada escenario. 
Un tercer grupo de sitios: Cardonal, La Bolsa, Los Viscos y Mortero Quebrado se encuentra en zonas de probabilidad positiva (aunque reducida). En este último caso, durante las tareas de campo que hemos realizado en el valle de Anfama no hemos evidenciado la presencia de la especie, y su baja presencia relativa permitiría pensar en un origen alóctono; sin embargo, el MDE no nos permitiría descartar su origen local en tiempo actual y pasado. Adicionalmente, podríamos considerar que la probabilidad de presencia de la especie a escala local no sería suficiente para abastecer a colectivos aldeanos amplios.

Un último grupo, compuesto por los sitios Casas Viejas, El Colorado, Soria 2 y Yánimas 1, se encuentra en sectores donde la probabilidad de ocurrencia es alta y cuyo aprovisionamiento se habría dado en sectores inmediatos (menos de $5 \mathrm{~km}$ ).

Sintéticamente, las evidencias de consumo de Geoffroea decorticans en sitios elevados de la puna y/o de las cumbres calchaquíes implicaría necesariamente el establecimiento de relaciones con sus vecinos vallistos y/o de las yungas. Los resultados refuerzan hipótesis ya planteadas de que el chañar integraría redes de circulación de recursos y/o personas, al igual que otros bienes que circularon por amplios sectores del área sudcalchaquí (Lazzari, 2010; Martel et al., 2017, entre otros). La presencia en sitios donde su origen sería extralocal o donde su obtención implicaría esfuerzos por fuera de lo cotidiano posibilita pensar en una intención marcada para obtenerlo y en la importancia asignada a la especie por grupos considerados tradicionalmente agropastoriles y sedentarios, no solo con fines utilitarios (e.g., leña, madera o alimento), sino también como adornos y ofrendas funerarias (Babot et al., 2008).

Lo antedicho implicaría relaciones permanentes, recurrentes y cíclicas entre los espacios habitados por la especie y las personas que bregaban por su obtención. Y también, como plantean Lorandi y Boixados (1988), que los sectores de chañarales (y algarrobales) pudieron ser "chawpi", es decir, lugares comunes de encuentro, de festejo y también de conflicto, cuyos derechos de explotación pudieron no siempre estar totalmente codificados.

Por otra parte, corresponde marcar que la probabilidad de presencia de la especie no es per se un indicador totalmente fiable de la presencia anual de frutos. Un aspecto importante a ser considerado en el análisis de este trabajo y en otros donde se apliquen MDE es la variabilidad en los caracteres florales y en la producción de pericarpios y semiIlas que puede exhibir una especie según el año y las condiciones climáticas en las que se encuentre, tal como se evidenció en Geoffroea decorticans (Eynard y Galetto, 1999), cebil (Anadenanthera colubrina var. Cebil [Vell.] Brenan; de Viana et al., 2014), algunas variedades de algarrobo (Prosopis alba Griseb. y Prosopis nigra [Griseb.] Hieron., Storni, 1942), entre otros.

Por ejemplo, se espera que aquellos ejemplares que se encontraran en los umbrales probabilísticos más bajos de ocurrencia produjeran una cantidad inferior de frutos que aquellos ubicados en sectores de alta probabilidad, o que en sectores que el MDE asigna a una baja probabilidad de ocurrencia no se desarrollara la especie, tal como es el caso del valle de Anfama en la actualidad. Adicionalmente, el chañar ha sido y es ampliamente valorado por su madera (i.e., Capparelli y Raffino, 1997; Marconetto, 2005), con lo cual pueden plantearse salvedades similares a su uso maderero, ya que el tamaño y volumen de los ejemplares varía en función de las características climáticas. Entonces, aun al obtenerse un valor de probabilidad de presencia de la especie para un sitio, no se puede asegurar que los recursos que brinda la planta hayan sido obtenidos efectivamente de tal lugar, debido a que desconocemos su fenología particular en las diferentes latitudes y en los diferentes climas.

\section{CONSIDERACIONES FINALES}

El objetivo de este trabajo fue mostrar las potencialidades inferenciales del MDE a partir de MaxEnt; esta herramienta puede ser aplicada para indagar la paleodistribución de especies vegetales y animales con un mayor grado de sustento analítico que la observación analógica. Se ejemplificó un uso posible de esta a partir del modelado de Geoffroea decorticans en tiempo actual y durante el Holoceno tardío en distintos sitios del área sudcalchaquí durante el primer milenio d.C.

Esperamos que las hipótesis planteadas dentro de la ejemplificación puedan ser contrastadas en un futuro próximo a partir de la ampliación de líneas de investigación disciplinar. Pensamos que el MDE puede integrarse a la batería de análisis habitualmente realizados dentro de esas líneas teóricas y perfeccionarse a partir de allí. 
Al respecto, esperamos poder ampliar los modelados incluyendo otras especies de uso/consumo frecuente y así obtener una mirada más integral sobre determinadas poblaciones y la movilidad de recursos. Consideramos importante la utilización de MDE para aproximarnos a las paleodistribuciones, pero como una herramienta susceptible de ser afinada a partir del conocimiento biológico intensivo de las especies.

Finalmente, reiteramos que es necesario trascender la subsunción de los recursos de recolección a narrativas marginales dentro de las lógicas de reproducción social en sociedades que ya habían desarrollado cultivos (Lema, 2008; Ortiz y Killian Galván, 2016). Para ello, es necesario perfeccionar herramientas analíticas que permitan adentrarnos en las implicancias profundas que tuvo la extracción de recursos.

\section{Agradecimientos}

A Rocío Molar, Julián Salazar y Matías Medina por su revisión del manuscrito original. A los colaboradores de las distintas campañas arqueológicas realizadas en Anfama. Centros de investigación: IEH (CONICET) e IMBIV (UNC - CONICET). A los evaluadores anónimos, que mejoraron las versiones originales del manuscrito; los errores y omisiones son nuestros.

\section{REFERENCIAS CITADAS}

Allouche, O., Tsoar, A., y Kadmon R. (2006). Assessing the accuracy of species distribution models: prevalence, kappa and the true skill statistic (TSS). Journal of Applied Ecology, 43, 1223-1232.

Alvarado-Serrano, D. F. y Knowles L. (2014). Ecological niche models in phylogeographic studies: applications, advances and precautions. Molecular Ecology Resources, 14, 233-248.

Anderson, R.P., Gómez-Laverde, M., y Peterson, A. (2002). Geographical distributions of spiny pocket mice in South America: insights from predictive models. Global Ecology and Biogeography, 11, 131-141.

Aschero, C. y Hocsman S. (2011). Arqueología de las ocupaciones cazadoras-recolectoras de fines del Holoceno medio de Antofagasta de la Sierra (Puna meridional argentina). Chungara, 43(especial), 393-411.
Babot, M. P. (2011). Cazadores-recolectores de los Andes centro-sur y procesamiento vegetal, Una discusión desde la Puna meridional argentina (Ca. 7.000-3.200 años A.P.). Chungara, 43(especial), 413-432.

Babot, P., González, L., Urquiza, S., Aguirre, M., Colaneri, M., Hocsman, S., y Haros, M. (2008). Dinámicas de formación y transformación de un entierro en el desierto puneño (Antofagasta de la Sierra, Puna Meridional Argentina). Intersecciones en Antropología, 10, 183-201.

Besio, L. (2017). Chañarales: espacios simbólicos de cura y peligro. Ensayo realizado sobre notas etnográficas con campesinos de comunidades huarpes en el departamento de Lavalle, al NE de la provincia de Mendoza (Argentina). Revista del Museo de Antropología, 10, 105-116.

Brown, J., Hill, D., Dolan, A., Carnaval, A. y Haywood, A. (2018). PaleoClim, high spatial resolution paleoclimate surfaces for global land areas. Scientific data, 5,180-254.

Calo, C. (2010). Plantas útiles y prácticas cotidianas entre los aldeanos al sur de los Valles Calchaquíes (600 a.C. 900 d.C.) [Tesis doctoral inédita. Universidad Nacional de La Plata].

Camps, G., Martínez-Meyer, E., Verga, A., Sérsic, A. y Cosacov, A. (2018). Genetic and climatic approaches reveal effects of Pleistocene refugia and climatic stability in an old giant of the Neotropical Dry Forest. Biological Journal of the Linnean Society, 125(2), 401-420.

Capparelli, A. y Raffino, R. (1997). La etnobotánica de El Shincal (Catamarca, Argentina) y su importancia para la Arqueología I: recursos combustibles y madereros. Parodiana, 10(1-2), 181-188.

Carrizo, J., Cano, S. y Soler Nixdorff, M. (1997). Recursos vegetales comestibles en el valle de Tafí durante el período Formativo: análisis arqueobotánico del sitio Casas Viejas-El Mollar (S Tuc Tav 2). En Actas del XII Congreso Nacional de Arqueología Argentina, Tomo I (pp. 65-69). Universidad Nacional de La Plata.

Cohen, J. (1960). A coefficient of agreement of nominal scales. Educational and Psychological Measurement, $20,37-46$.

de Viana, M., Giamminola, E., Russo, R. y Ciaccio, M. (2014). Morphology and genetics of Anadenanthera colubrina var. cebil (Fabaceae) tree from Salta (Northwestern Argentina). Revista de biología tropical, 62(2), 757-767.

Demaio, P. H., Karlin, U.O. y Medina, M. (2002). Árboles nativos del centro de Argentina. L.O.L.A. 
Elith, J. y Leathwick, J. R. (2009). Species Distribution Models: Ecological Explanation and Prediction Across Space and Time. Annual Review of Ecology, Evolution, and Systematics, 40(1), 677-697.

Elith, J., Kearney, M. y Phillips, S. (2010). The art of modelling range-shifting species. Methods in Ecology and Evolution, 1, 330-342.

Eynard, C. y Galetto, L. (1999). Estructura floral y variabilidad intraespecífica de Geoffroea decorticans (Fabaceae). Darwiniana, 37, 219-228.

Farberman, J. (2006). Recolección, economía campesina y representaciones de los montaraces en Santiago del Estero, siglos XVI a XIX. Prohistoria, 10, 11-26.

Fielding, A. y Bell, J. (1997). A review of methods for the assessment of prediction errors in conservation presence/ absence models. Environmental Conservation, 24, 38-49.

Fordham, D., Saltré, F., Haythorne, S., Wigley, T., OttoBliesner, B., Chan, K. y Brook, B. (2017). PaleoView: a tool for generating continuous climate projections spanning the last 21000 years at regional and global scales. Ecography, 40, 1348-1358.

Gómez Augier, J. y Caria, M. (2012). Los paleoambientes y los procesos culturales en el Noroeste Argentino: una aproximación desde la arqueología de Tucumán. Acta Geológica, 24, 80-97.

Grana, L., Tchilinguirian, P., Olivera, D., Laprida, C. y Maidana, N. (2016). Síntesis paleoambiental en Antofagasta de la Sierra: heterogeneidad ambiental y ocupaciones humanas en los últimos 7200 años cal AP. Intersecciones en Antropología [vol. Especial], 4, 19-32.

Ireland, H. y Pennington, R. (1999). A revision of Geoffroea (Leguminosae-Papilionoideae). Edinburgh Journal of Botany, 56(3), 329-347.

Karger, D., Conrad, O., Böhner, J., Kawohl, T., Kreft, H., Soria-Auza, R., Zimmermann N., Linder H. y Kessler, M. (2017a). Climatologies at high resolution for the earth's land surface areas. Scientific Data, 4, 170-122.

Karger, D., Conrad, O., Böhner, J., Kawohl, T., Kreft, H., Soria-Auza, R., Zimmermann N., Linder H. y Kessler, M. (2017b). Data from: Climatologies at high resolution for the earth's land surface areas. https://datadryad.org/stash/

Kelly, R. (1992). Mobility/Sedentism: Concepts, Archaeological Measures, and Effects. Annual Review of Anthropology, 21, 43-66.

Korstanje, M. A. y Würschmidt A. (1999). Producir y recolectar en los valles altos del NOA: Los Viscos como caso de estudio. En C. Aschero, A. Korstanje y P. Vuoto (eds.), Los tres reinos: prácticas de recolección en el cono sur de América (pp. 151-160). Instituto de Arqueología y Museo.
Kulemeyer, J., Lupo, L., Madozzo, M., Cruz, A., Cuenya P., Maloberti, M., Cortés, G. y Korstanje, A. (2013). Desarrollo del Paisaje Holoceno en la Cuenca de El Bolsón: gente y ambiente en procesos de cambio y estabilidad. Diálogo Andino, 41, 25-44.

Lazzari, M. (2010). Landscapes of circulation in NW Argentina: The workings of obsidian and ceramics during the first millenium AD. En A. Bauer y A. Agbe-Davies (eds.), Social archeologies of trade and exchange, exploring relationships among people, places and things (pp. 49-68). Left Coast Press.

Lema, V. (2008). ¿De qué hablamos cuando hablamos de domesticación vegetal en el NOA? Revisión de antiguas propuestas bajo nuevas perspectivas teóricas. En S. Archila, M. Giovannetti y V. Lema (eds.), Arqueobotánica y Teoría Arqueológica, discusiones desde Suramérica (pp. 97-127). Universidad de los Andes.

Lema, V., Capparelli, A. y Martínez, A. (2012). Las vías del algarrobo: Antiguas preparaciones culinarias en el Noroeste argentino. En M. P. Babot, M. Marschoff y F. Pazzarelli (eds.), Las manos en la masa: arqueologías, antropologías e historias de la alimentación en Suramérica (pp. 639-667). Universidad Nacional de Córdoba.

López Campeny, S. y Escola, P. (2007). Un verde horizonte en el desierto: producción de cuentas minerales en ámbitos domésticos de sitios agropastoriles. Antofagasta de la Sierra (Puna Meridional Argentina). En A. Nielsen, M. C. Rivolta, V. Seldes, M. Vázquez y P. Mercolli (comps.), Producción y circulación prehispánicas de bienes en el sur Andino (pp. 225-258). Brujas.

Lynch, S. y Rocek, T. (2019). The Dunlap-Salazar site lithic sources and highland pithouse-period mobility in the Jornada Mogollon of south-central New México. En T. Rocek y N. Kenmotsu (eds.), Late prehistoric huntergatherers and farmers of the Jornada Mogollon (pp. 124-136). University Press of Colorado.

Marconetto, B. (2005). Recursos forestales y el proceso de diferenciación social en tiempos prehispánicos en el valle del Ambato, provincia de Catamarca [Tesis doctoral inédita. Universidad Nacional de La Plata].

Martel, A., Zamora, D. y Lépori, M. (2017). Tráfico y movilidad caravanera en la Puna catamarqueña. Una mirada internodal. Estudios Atacameños, 56, 197-223.

Miguez, G. y Caria, M. (2015). Paisajes y prácticas sociales en las selvas meridionales de la provincia de Tucumán ( ${ }^{\circ}$ milenio d.C.). En M. Korstanje, M. Lazzari, M. Basile, F. Bugliani, V. Lema, L. Pereyra y M. Quesada (eds.), Crónicas materiales precolombinas, Arqueología de los primeros poblados del Noroeste Argentino (pp. 111-148). Sociedad Argentina de Antropología. 
Molar, R. (2014). Procesar, consumir y construir.

Alimentación y reproducción de la cotidianeidad en sociedades aldeanas tempranas del primer milenio D.C, en el Valle de Tafí [Tesis de grado inédita. Universidad Nacional de Córdoba].

Muscarella, R., Galante, P., Soley-Guardia, M., Boria, R., Kass, R. J., Uriarte, M. y Anderson, R. P. (2014). ENMeval: An R package for conducting spatially independent evaluations and estimating optimal model complexity for Maxent ecological niche models. Methods in Ecology and Evolution, 5, 1198-1205.

Muscarella R., Galante, P., Soley-Guardia., M., Boria, R., Kass, J., Uriarte, M. y Anderson, R. (2017). Package ENMeval. Automated Runs and Evaluations of Ecological Niche Models. Version 0.2.2. https://cran.rproject.org $/$ package $=$ ENMeval

Noli, E. (1998). Algarrobo, maíz y vacas. Los pueblos indios de San Miguel del Tucumán y la introducción de ganados europeos (1600-1630). Mundo de Antes, 1, 31-67.

Oliszewski, N., Martínez, J., Di Lullo, E., Gramajo Bühler, C., Arreguez, G., Cruz, H.,...Srur, M. (2015). Contribuciones al estudio de sociedades aldeanas en el Noroeste argentino: El caso de la Quebrada de los Corrales (El Infiernillo, Tucumán). En M. Korstanje, M. Lazzari, M. Basile, F. Bugliani, V. Lema, L. Pereyra y M. Quesada (Eds.), Crónicas materiales precolombinas, Arqueología de los primeros poblados del Noroeste Argentino (pp. 51-80). Sociedad Argentina de Antropología.

Oliszewski, N. (2017). Las aldeas "patrón Tafí" del sur de cumbres Calchaquíes y norte del sistema del Aconquija. Comechingonia, 21, 205-232.

Orrabalis, C., Gorostegui, H., Calandri, E. y Guzmán, C. (2013). Parámetros funcionales y contenido de glucosa en harinas obtenidas de fruto maduro de "chañar" (Geoffroea decorticans) de la zona semiárida y árida de la provincia de Formosa. Multequina, 22, 15-22.

Ortiz, M. G., y Killian Galván, V. (2016). El consumo como vía para comprender economías mixtas. Su aplicación al sur del valle de San Francisco, región pedemontana de Jujuy (Argentina). En S. Alconini (ed.), Entre la vertiente tropical y los valles Sociedades regionales e interacción prehispánicas en los Andes centro-sur (pp. 263-282). Plural.

Osorio-Olvera L., Barve, V., Barve, N., Soberón, J. y Falconi, M. (2018). Ntbox: From getting biodiversity data to evaluating species distribution models in a friendly GUI environment. R package version 0.2.5.4. https://github.com/luismurao/ntbox
Owens, H., Campbell, L., Dornak, L., Saupe, E., Barve, N., Soberón, J.,...Peterson, A. (2013). Constraints on interpretation of ecological niche models by limited environmental ranges on calibration areas. Ecological Modelling, 263, 10-18.

Oyarzabal M., Clavijo, J., Oakley, L., Biganzoli, F., Tognetti, P., Barberis, I.,...León, R. (2018). Unidades de vegetación de la Argentina. Ecología Austral, 28, 40-63.

Palamarczuk, V., Raíces Montero, C., García, M. C., Molina, C., Daizo, M. B., Petrucci, N.,...Greco, C. (2016). El Colorado. Avances en las investigaciones arqueológicas en una localidad del sur de Yocavil, Catamarca, Argentina. En Actas del XIX CNAA (pp. 432-433). Universidad Nacional de Tucumán.

Peterson A., Papes, M. y Eaton, M. (2007). Transferability and model evaluation in ecological niche modeling: a comparison of GARP and Maxent. Ecography, 30, 550-560.

Peterson A., Papes, M. y Eaton, M. (2008). Rethinking receiver operating characteristic analysis applications in ecological niche modeling. Ecological Modelling, 213, 63-72.

Peterson A., Soberón, J., Pearson, R., Anderson, R., Martínez-Meyer, E., Nakamura M. y Araújo, M. (2011). Ecological niches and geographic distributions. Princeton University Press.

Petrucci, N. y Spano, R. (2019). Arqueobotánica del sitio temprano Soria 2 (Catamarca, Argentina). Boletín de la $S A B, 54(1), 137-154$.

Phillips, S., Dudík, M. y Shapire, R. (2004). A maximum entropy approach to species distribution modeling. En R. Greiner y D. Schuurmans (eds.), Proc. 21st Int. Conf. on Machine Learning (pp. 655-662). ACM press.

Phillips S. J., Anderson, R. P. y Schapire, R. E. (2006). Maximum entropy modeling of species geographic distributions. Ecological Modelling, 190, 231-259.

Pochettino, M. L., y Scattolin, M. C. (1991). Identificación y significado de frutos y semillas carbonizados de sitios arqueológicos formativos de la ladera occidental del Aconquija (Catamarca, República Argentina). Revista del Museo de La Plata, 9, 169-182.

Politis, G., Prates, L., Merino, M. y Tognelli, M. (2011). Distribution parameters of guanaco (Lama guanicoe), pampas deer (Ozotoceros bezoarticus) and marsh deer (Blastocerus dichotomus) in Central Argentina: Archaeological and paleoenvironmental implications. Journal of Archaeological Science, 38(7), 1405-1416.

Politis, G., Domic, A., Bonomo, M. y Capriles, J. (2018). Modelamiento de los parámetros ambientales de la distribución de sitios Goya-Malabrigo mediante 
Maxent. En G. Politis y M. Bonomo (eds.), GoyaMalabrigo: arqueología de una sociedad indígena del Noreste argentino (pp. 289-305). Universidad Nacional del Centro de la Provincia de Buenos Aires.

Rocek, T. (1996). Sedentism and mobility in the Southwest. En P. Fish y J. Reid (eds.), Interpreting southwestern diversity: underlying principles and overarching patterns (pp. 17-25). Arizona State University.

Salazar, J. y Molar, R. (2017). Estudio comparativo de dos sitios aldeanos del primer milenio d.C. en Tucumán, Argentina. Comechingonia, 21, 123-148.

Scattolin, C. (2007). Santa María antes del año mil. Fechas y materiales para una historia cultural. En V. Williams, B. Ventura, M. Callegari y H. Yacobaccio (eds.), Sociedades Precolombinas Surandinas. Temporalidad, Interacción y Dinámica Cultural del NOA (pp. 203220). T.I.A. NOA y Andes Centro-Sur.

Soberón J. y Peterson, A. (2005). Interpretation of models of fundamental ecological niches and species' distributional areas. Biodiversity Informatics, 2, 1-10.

Storni, J. (1942). Bromatología indígena, solución precolombiana del problema alimenticio. Universidad Nacional de Tucumán.

Tortorelli, L. (1956). Maderas y bosques argentinos, tomo 1. Orientación gráfica.
Yacobaccio, H. y Korstanje, M. (2007). Los procesos de domesticación vegetal y animal. Un aporte a la discusión argentina en los últimos 70 años. Relaciones de la Sociedad Argentina de Antropología, XXXII, 191-215.

\section{NOTAS}

1.- Se considera dentro de la denominación a los faldeos y piedemontes orientales de las cumbres calchaquíes, a los vaIles de La Ciénega, Tafí, Yocavil, El Cajón, y al sector puneño de Antofagasta de la Sierra. Las poblaciones de estos sectores mantuvieron una fluida circulación de materiales, ideas y personas a lo largo del primer milenio (Scattolin, 2007; Babot et al., 2008; Lazzari, 2010; Gómez Augier y Caria, 2012; Oliszewski, 2017; Salazar y Molar, 2017, entre otros).

2.- Para ahondar en la teoría y práctica de los MDE se puede consultar Elith y Leathwick (2009), Peterson et al. (2011). En tanto que mayores aspectos sobre el funcionamiento y práctica de MaxEnt pueden leerse en Phillips et al. (2006), Muscarella et al. (2014), entre otros.

3.- Disponible en http://paleoclim.org/

4.- Este lapso es el máximo grado de resolución temporal que existe actualmente para el modelado a partir de datos paleoclimáticos, lo cual permite la realización de modelos aproximados con las limitaciones y salvedades asociadas a trabajar con promedios en rangos amplios.

5.- GBIF.org; descarga de ocurrencias en https://doi. org/10.15468/dl.icrkhf 
